\title{
Mapping the Content Structure of Online Diabetes Support Group Activity on Facebook
}

Zörgő, S., Jeney, A., Csajbók-Veres, K., Mkhitaryan, S., Susánszky, A.

This paper has been published in the proceedings of the International Conference on Quantitative Ethnography (2021)

\section{Citation information:}

2022 Zörgő, S., Jeney, A., Csajbók-Veres, K., Mkhitaryan, S., Susánszky, A. Mapping the Content Structure of Online Diabetes Support Group Activity on Facebook. In: Advances in Quantitative Ethnography. Communications in Computer and Information Science, Vol 1522. Eds. Wasson B and Zörgö S., pp 221-236. Cham, Switzerland: Springer Nature. 


\section{Mapping the Content Structure of Online Diabetes Support Group Activity on Facebook}

Zörgő, S., Jeney, A., Csajbók-Veres, K., Mkhitaryan, S., Susánszky, A.

This paper has been accepted to the International Conference on Quantitative Ethnography (2021) and will be published in its proceedings. Please check back here in December 2021 for precise citation. 


\title{
Mapping the Content Structure of Online Diabetes Support Group Activity on Facebook
}

\author{
凶Szilvia Zörgö1 [0000-0002-6916-2097], Anna Jeney 2 [0000-0002-6037-9505], \\ Krisztina Csajbók-Veres ${ }^{3}$, Samvel Mkhitaryan ${ }^{[0000-0003-0667-0542]}$ and \\ Anna Susánszky 5 [0000-0002-4518-1314] \\ ${ }^{1,5}$ Institute of Behavioral Sciences, Semmelweis University, Budapest, Hungary \\ zorgoszilvialgmail.com \\ ${ }^{2}$ Department of Cultural Anthropology, Eötvös Loránd University, Budapest, Hun- \\ gary \\ ${ }^{3}$ Center for Intercultural Psychology and Education, Eötvös Loránd University, Bu- \\ dapest, Hungary \\ ${ }^{4}$ Department of Health Promotion, CAPHRI Maastricht University, Maastricht, The \\ Netherlands
}

\begin{abstract}
Diabetes is one of the most prevalent chronic diseases and necessitates ongoing self-management to control symptoms. People with diabetes (PWD) express various needs, which are often met in online support groups. Our objectives were to examine related discourse in Hungarian Facebook groups in order to map the content and structure of information PWD are sharing. We aimed to juxtapose our findings with the functionality of eHealth tools available to Hungarian patients to understand what these tools may be lacking. We extracted a total of 200 threads from two public self-help groups. Codes were developed inductively and applied to the entire corpus with the Interface for the Reproducible Open Coding Kit. We used Epistemic Network Analysis to model our data. The mean network of the entire dataset indicates that discourse was chiefly centered on providing help regarding lifestyle-related issues, especially diet. Critical facets of discourse included the need by experienced PWD to provide guidance to the newly diagnosed, share "street-level" knowledge on diabetes, and ensure a sense of support and community. Existing eHealth tools could be supplemented with an app that automatically downloads blood glucose meter measurements, warns patients about predicted high glucose levels, enables location-based information exchange, and promotes social support in adhering to diet and exercise. Ideally, this app would connect patients with their physicians and/or dietitians.
\end{abstract}

Keywords: Reproducible Open Coding Kit (ROCK) - Epistemic Network Analysis (ENA) $\cdot$ Diabetes $\cdot$ Social media $\cdot$ eHealth 


\section{Introduction}

Diabetes is currently one of the most prevalent chronic diseases; the number of people with diabetes (PWD) in the US has tripled in the past 20 years with figures also increasing in Europe [1]. Regardless of type, diabetes requires ongoing self-management to control symptoms and negative effects through careful attention to nutrition, sleep, exercise, etc., and includes performing various daily tasks, such as checking blood glucose levels and adhering to medical regimens. PWD may spend up to 8000 hours per year managing their disease outside of the medical setting [2].

Patients report experiencing diabetes-related stigma, exclusion, rejection, blame, and negative judgement, which may lead PWD to feel isolated from others [3]. Due to these experiences, coupled with the need to navigate the intricacies of self-management, PWD express strong preferences for peer support [4], which demand is often met in online support groups, collectively referred to as the Diabetes Online Community (DOC). Via these forums, patients can interact asynchronously to acquire and share information e.g., in the domains of nutrition and treatment options.

Core activities in such online social support networks include obtaining information about the disease, sharing personal opinions and experiences, receiving emotional support for distress arising from demanding self-care regimes and medical complications resulting from the disease [5]. Ubiquitous availability, anonymity, selective disclosure, and social networking present ample opportunities for sharing information, being validated, as well as receiving advice and empathy [5].

Oser et al. direct attention to other motivations that drive participation in DOCs: patients may perceive a lack of "real-world" knowledge (e.g., travel advice for PWD) among healthcare providers or system contributors, patients may seek to educate themselves to practice health literacy and further their knowledge, and they may have unmet psychosocial needs, which they prefer to address with social support outside of the medical setting [2].

Several authors have discussed difficulties in judging the veracity of disease-related information shared in such online support groups, where information gains credibility through social validation: consensus is reached through comments and other forms of engagement [5]. For example, Weitzman et al. warn that disclosed information may frequently be aligned with diabetes science, but group discourse may have gaps in medical disclaimer use and critical moderation [5]. Albeit the extent to which DOCs influence clinical outcomes is unclear [2,5], their effects on well-being are less disputed. Many studies report improved well-being among DOC participants: a reduction in feelings of isolation [6,7], an increased sense of belonging [8] and empowerment [9], as well as increased self-efficacy in diabetes self-management $[1,6]$.

DOC discourse provides crucial insight into how patients interact with information and with each other, their psychosocial and clinical needs, as well as their disease-related behavior, especially regarding domains where conventional care is perceived to be lacking [1]. Analyzing such discourse lays the foundation for developing eHealth systems to better serve the psychosocial needs of PWD and to help them cope with daily routines involved in managing the disease. 
Our objectives were to examine DOC discourse in Hungarian diabetes-related Facebook groups in order to map the content and structure of information that patients are sharing.

RQ1: What kind of content is shared in patient self-help groups and how do patients interact with these?

RQ2: How are these pieces of content related to each other?

Findings were juxtaposed to the functionality of eHealth tools available to Hungarian patients to understand what these tools may be lacking and what direction further development aimed at aiding diabetes self-management may take.

\section{Previous Approaches to Mapping Shared Content}

Research of diabetes-related social media groups includes studies conducted on major platforms, such as Twitter, Instagram, Facebook, and personal blogs. According to a recent review of such works [2], authors have thus far chiefly focused on post content (subject matter of posted content and related comments), message features (type of content, e.g.: text, picture, video), and engagement (number of likes, comments, shares). In some cases, additional information has also been scrutinized, such as number of group members, number of new members in past 30 days, frequency of posts, and months in operation (e.g.: [1]).

In most cases, authors were interested in what subject and type of content received the most engagement and used multivariable logistic regressions to evaluate associations among various group characteristics. For example, Gabarron et al. employed seven discourse codes (1: health education, 2: research and innovation, 3: diabetes-related technology, 4: interviews and personal stories, 5: awareness days and celebrations, 6: recipes and food-related information, and 7: miscellaneous) to deductively code posts on three social media platforms of the Norwegian Diabetes Association [6]. Comments under posts were not coded, and each post $(\mathrm{N}=1449)$ could only receive one discourse code. The authors employed binomial regression analyses of the effects of content topic (discourse codes), social media channel (Twitter, Facebook, Instagram), and post features (emojis, picture/text/video) as predictors of user engagement. They concluded that "interviews and personal stories" received the most, while recipes and food-related information received the least engagement; furthermore, videos and emojis were the most engaging post features. Although these are important details concerning the relationship between post subject and engagement, these analyses do not lend deeper insight into the actual content that is being shared, with what purpose it is shared, and how participants meaningfully engage in knowledge construction and/or social validation.

Another common feature of publications regarding the content shared by DOCs is providing code frequencies or the relative frequencies of codes as percentages of total posts coded. For example, one of Greene et al.'s key findings was that "Providing information" was the most frequent code in their dataset (65.7\%), followed by "Support" (28.8\%) and "Advertisements" (26.7\%). Yet, to what these codes relate is unclear, e.g., regarding what aspect of diabetes self-management (e.g., blood glucose measurement, nutrition, exercise, psychosocial distress) is information or support provided? Albeit code frequencies are valuable indicators of relevance regarding coded content, 
individual occurrences of codes tell the analyst little about how meaning is constructed within posts and threads, and how codes interact with each other to form substance.

Zhang et al. took a more detailed approach by inductively developing hierarchical codes and reporting the distribution of child codes within their parents, not merely the relative frequency of the parent codes [10]. For example, the authors state that $74 \%$ of all examined DOC messages (posts and comments, $\mathrm{N}=1352$ ) were related to the parent code "Information" (eliciting or providing information), and reported that $57.9 \%$ of that parent code consisted of messages tagged as "Medical info" and 20.1\% as "Lifestyle" (ibid). Although it is clear from the publication that "Emotion" was the second most frequent code in the narrative corpus, the reader does not know how this code is related to "Community-building", the last of three parent codes, nor is the reader privy to how these parent and child codes relate to each other in the individual "messages" or the entire corpus. Hierarchical code structures may be a solution to some limitations of coding-and-counting analyses, but it does not address the problem of temporality in discourse data and its socio-cognitive effects [11].

Rus et al. employed multi-level negative binomial regression analyses of message features as predictors of user engagement in diabetes-related Facebook pages. The coding scheme was adopted from the National Cancer Institute's guidelines on health communication elements. Regression analyses were performed on coding categories regarding both discourse content and message features; content within the post text (e.g., causal information, control information, timeline, consequence information, negative/positive affect) was not distinguished conceptually from message features, such as the presence or absence of imagery or external links. Results show that "Imagery $\mathrm{x}$ Control Information" and "Imagery x Consequence Information" emerged as significant interactions predictive of user engagement. [12] This approach highlights the importance of interaction among codes to gain more insight on how message features relate to user engagement. Yet the reader is only privy to a small subset of interactions, while crucial connections could remain unearthed.

Albeit coding-and-counting methods may be useful in describing basic characteristics of the coded narrative corpus, they often disregard or attempt to control for phenomena that are essential to meaning construction. Treating discourse codes and/or message features as independent entities assumes that meaning-making by an individual (e.g., in a post) or by a group of individuals (e.g., in a thread) can be modelled and understood by inspecting frequency alone without reference to other codes. Codingand-counting reflects code frequencies in isolation, but do not take into account how those codes are connected to other codes to convey meaning, and how participants are engaging with that meaning and with each other to co-produce knowledge. Hence, identifying message features that predict high levels of engagement tell us little about the content of the message, and reporting code frequencies within a corpus does not unveil the underlying interdependent nature of discourse elements. Rarely does a single code (similarly to a single word) carry meaning in isolation; meaning emerges from the interplay of linguistic and conceptual elements, as well as their temporality [11]. Rather than through codes and frequencies, meaning is more accurately captured via code cooccurrences and code constellations.

Epistemic Network Analysis (ENA) generates networks that model and describe code interactions within units of analysis, which can be, for instance, individual 
contributors, posts, threads, or entire narrative corpuses. Because text is segmented and code co-occurrences are accumulated accordingly, ENA can account for temporality within verbal data. Networks are constructed based on adjacency matrices capturing code connection counts for each unit of analysis, and a cumulative adjacency matrix aggregates matrices for each unit of analysis. These are represented as vectors, where columns in this matrix correspond to code pairs and rows to a point in high-dimensional space. Spherical normalization is performed to account for the fact that the length of the vectors is potentially affected by the length of narratives in each unit. In this process, each vector is divided by its own length, resulting in a normalized vector quantifying the relative frequencies of code co-occurrence, which is hence expressed as connection strengths falling between zero and one. ENA then performs dimensional reduction via singular value decomposition (SVD), a technique commonly applied in principle component analysis. The resulting space expresses the differences among units, whose networks receive two, coordinated representations: 1) a projected point or "ENA score" (the location of a unit's network in the low-dimensional projected space) and 2) a weighted network graph (nodes correspond to the codes, edges represent the relative frequency of connection between two codes). For a more detailed explanation of the mathematics of ENA, see [13]. In the present study, we employed ENA to simultaneously harness the explanatory power of qualitative content analysis and describe coded and quantified DOC discourse by modelling the relationship among codes, as well as their relative frequency.

\section{$3 \quad$ Methods}

\subsection{Sampling}

Facebook is one of the most commonly used social networking websites for connecting people with chronic illnesses [1]; on average, 1.59 billion active users engage with/produce content daily [5]. Of the available major social media platforms, Facebook use far outweighs any other (Twitter, Instagram, etc.) in Hungary [14]. Thus, we chose Facebook to explore user-generated content in Hungarian DOCs. The "groups" feature is designated for dedicated communication about shared interests; we used the search function to identify diabetes-related public groups.

We employed purposive, homogeneous sampling; our inclusion criteria were as follows: public Facebook self-help group founded and populated by adult patients of diabetes; language is Hungarian; minimum 100 members; post frequency is minimum once a week; and last post occurred within a week. Our exclusion criteria were: group is private; group is explicitly tied to a religion, an organization, a hospital, or a university; group was founded by and/or is maintained by a health practitioner, e.g., physician, nurse, or naturopath; group is organized around a single healthcare product or product line; group is aimed at healthy living in general. Two researchers performed the search for possible groups to be included in the sample.

The majority of such groups were private and required acceptance into the group to view shared content. As user consent should have been obtained from all members 
within private groups to investigate such discourse (including those no longer present in the group but contributing to examined content), we decided to analyze the content of public groups only. Two groups fit our inclusion criteria; these two public groups constituted "cases", i.e. data providers in our study. Group 1 had 2800 members at the time of preregistration, Group 2 had 1900 members. Neither researcher was an administrator or member of either group that was reviewed. Scraped data could not pre-date March 13, 2020, the beginning of the first COVID-related lockdown in Hungary; we implemented this restriction as we surmised that pre- and post-COVID discourse (and hence expressed needs) would differ greatly and should not be included into the same sample.

\subsection{Data Extraction and Segmentation}

We extracted 100 threads (posts and their first-level comments) from both data providers $(\mathrm{N}=200)$; a thread constituted a source in our study. Data was scraped on March $16^{\text {th }}, 2021$ from the website and placed into individual sources (text files) containing a thread each. Only text data was collected; engagement data and message features, such as whether it contained photos/videos were not extracted because our research questions addressed text content only. We were not interested in examining what content generated the most engagement; every thread was considered a vital constituent of DOC discourse. Sometimes, a post consisted of only a picture and no comments; in this case, the source would be empty after scraping. To compensate for empty sources, we manually extracted additional threads $(n=63)$ in chronological order.

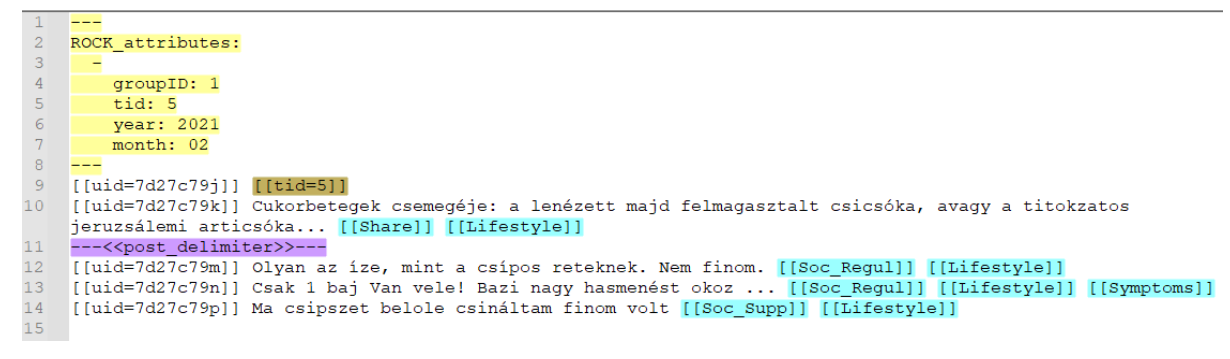

Figure 1. Example of a coded and segmented source in adherence to the Reproducible Open Coding Kit (ROCK) standard. Attributes are highlighted in yellow; the thread identifier is in green; discourse codes are in teal; and segmentation is highlighted in purple.

In the extraction process, sources were formatted to delimit post from comments and both post and each subsequent comment were separated by a newline character. Thus, our lowest level of segmentation (utterance) was a turn-of-talk (post or comment), a post-delimiter constituted mid-level segmentation, and all threads were housed in separate text files. Every utterance received a unique utterance identifier (uid) and every thread received a unique thread identifier (tid). Our dataset contained a total of 1333 utterances. Uids were generated using the Reproducible Open Coding Kit (ROCK) R 
package and all of our coding and segmentation adheres to the ROCK standard [15]. All sources were anonymized prior to coding and analysis.

\subsection{Code Development}

Discourse codes were developed inductively in several stages. We began by four researchers free-coding 10 threads and developing a preliminary codebook. Subsequently, these four preliminary codebooks were triangulated to create a tentative codebook, which was employed by the same four researchers to deductively code an additional 10 threads. Following this deductive coding stage, the four researchers triangulated their coded data and refined the codebook to create the final version. We juxtaposed the final version of our codebook to discourse codes we extracted from a precursory search in DOC-related research published between 2018 and 2021. We triangulated our codes with those salient in the literature, yet, because none of these articles provided their codebooks as supplementary information (e.g., URL to a repository), we were only privy to code labels and often (but not always) to short descriptions of utilized codes. Even though we found substantial overlap and similarity among these codes and ours, because identical code labels may refer to vastly different constructs [16], we cannot assert with confidence that we are measuring the same phenomena. Our final code structure contained three parent and 16 child codes. Table 1 displays our code labels and a description for each, as well as our Inter-rater reliability (IRR) scores.

Table 1: Final code structure and code descriptions and Inter-rater reliability (IRR) computed with Cohen's kappa $(\kappa)$ for the two pairs of raters

\begin{tabular}{|c|c|c|c|}
\hline PARENT CODE & CHILD CODE & КАРPA & DESCRIPTION \\
\hline \multirow{5}{*}{ ACtIVITy } & Ask4Help & 1 & Eliciting the opinions or experiences of others in the group \\
\hline & GiveHelp & 0.733 & $\begin{array}{l}\text { Stating an opinion, own experience, recommendations in response } \\
\text { to a demand }\end{array}$ \\
\hline & Soc_Supp & 0.733 & $\begin{array}{l}\text { Comforting, encouragement, congratulating, celebrating, } \\
\text { reinforcing }\end{array}$ \\
\hline & Soc_Regul & 0.733 & $\begin{array}{l}\text { Assessing the quality and reliability of shared information; } \\
\text { correcting, warning others about fraud or danger, negotiating } \\
\text { validity of information }\end{array}$ \\
\hline & Share & 0.845 & Unsolicited sharing of personal information or external content \\
\hline \multirow{2}{*}{ APPRAISAL } & Dissat & 0.727 & \multirow{2}{*}{$\begin{array}{l}\text { Satisfaction or dissatisfaction with a specific doctor, } \\
\text { pharmaceutical, or the healthcare system in general }\end{array}$} \\
\hline & Satisfact & 0.789 & \\
\hline \multirow{9}{*}{ CONTENT } & Instruments & 0.775 & Tools used in diabetes care (e.g., blood glucose meter) \\
\hline & Lifestyle & 0.842 & Recipes, healthy food, changes in body weight, exercise \\
\hline & Healthcare & 0.831 & Specific physician or the healthcare system in general \\
\hline & Pharma & 0.696 & Insulin and strictly diabetes-related pharma products \\
\hline & COVID & 0.789 & Vaccine, disease, risk estimation \\
\hline & TestValues & 0.776 & $\begin{array}{l}\text { Disclosing blood glucose level, various parameters, or biomedical } \\
\text { test result }\end{array}$ \\
\hline & Products & 0.697 & $\begin{array}{l}\text { Any prescription or over-the-counter drug, home remedy that } \\
\text { is not part of conventional diabetes care }\end{array}$ \\
\hline & Trade & 0.726 & Insulin-trade and barter of other diabetes-related drugs, products \\
\hline & Symptoms & 0.723 & $\begin{array}{l}\text { Side-effects of conventional or non-conventional diabetes care, } \\
\text { symptoms of diabetes or comorbidities }\end{array}$ \\
\hline
\end{tabular}




\subsection{Coding}

Codes were split among four researchers; a pair of raters "specialized" in the parent code Content and another pair of raters focused on Activity \& Appraisal. To calculate IRR, we computed Cohen's kappa $(\kappa)$ on $3 \%$ of the data $\left(n_{\text {utterances }}=40\right)$ for the codes designated to the two pairs of raters. For each of the 16 codes, the rate of agreement was above a minimum kappa threshold of 0.65 [17].

Subsequently, we employed the Interface for the Reproducible Open Coding Kit (iROCK) to deductively code the entire narrative corpus. Coding was performed on the level of utterances; one utterance could receive any number of codes. Within the pairs, each individual coded 100 threads; the two coded versions of sources (threads) were then merged with the ROCK R package. Attributes (relevant characteristics of threads) were added to the merged text files; we logged the following attributes for each thread: group ID (case 1 or case 2), thread ID (tid), and date (year and month). Upon completing coding, we parsed all 200 sources with the ROCK package and exported them into a csv file constituting our qualitative data table. In this dataframe, each row contained an utterance and every column represented a variable (attributes and discourse codes). Attributes were displayed in categorical form, discourse codes in binary form.

\subsection{Analysis}

We uploaded our qualitative data table into the Epistemic Network Analysis webtool to generate networks. After exploring various model parameters and iterating between the networks and coded data, we decided to employ a weighted whole conversation stanza window to accumulate code co-occurrences.

Table 2: Parameters of networks generated with Epistemic Network Analysis (ENA)

\begin{tabular}{l|l} 
UNIT & GroupID $>$ tid \\
\hline CONVERSATION & GroupID $>$ tid $>$ Post_Delimiter \\
\hline STANZA WINDOW SIZE & (Weighted) Whole conversation \\
\hline CODES & Activity, Appraisal, Content codes \\
\hline PROJECTION & MR1: $5.3 \%$, SVD2: $10 \%$
\end{tabular}

Our study was preregistered at Open Science Framework Registries. Our employed $\mathrm{R}$ scripts, detailed codebook, disclosed results, and extra visualizations can be openly accessed at our public repository: https://osf.io/7q65c/. This project constitutes part of a larger investigation entitled "E-patients and e-physicians in Hungary: The role and opportunities of digital health solutions in the healthcare system", a grant awarded by the National Research, Development and Innovation Office, Hungary. The ethics permit was issued by Semmelweis University Regional and Institutional Committee of Science and Research Ethics (SE RKEB: IV/10924-1/2020/EKU). Quotes from coded threads are disclosed in italics and quotation marks, and are followed by the thread identifier in parentheses; all quotes were translated from Hungarian by the authors. 


\section{$4 \quad$ Results}

\subsection{General Discourse Content and Structure}

The most prominent codes in the mean network of the entire dataset were GiveHelp and Lifestyle, which exhibited strong connections to each other, denoting instances of patients discussing diet-related issues, exchanging recipes, and conversing about weightrelated questions. Patients most frequently solicited help from others regarding Lifestyle (e.g., content and timing of meals), Products (e.g., herbs, OTC products, complementary medicine), and Pharma (pharmacological products used in conventional diabetes care). Help was provided in these domains, as well as regarding the interpretation of Symptoms (e.g., the normalcy or frequency of pain and various sensations, weight gain).

Shares in posts and replies were almost exclusively about Lifestyle, namely participants disclosing what they had for a meal; blood glucose levels were also frequently shared without any accompanying information. The marked co-occurrence of Lifestyle and Social Support indicates inter-member positive feedback and encouragement concerning diet, and comforting regarding changes in body weight. Aside from welcoming new members into the group and greeting each other on national holidays, Social Support also manifested in the normalization of diabetes-related Symptoms, and encouragement concerning blood glucose levels (TestValues). There was a strong connection among GiveHelp, Pharma, and Symptoms, indicating discourse around insulin use, types of diabetes medication, and experienced or anticipated side-effects of these. The most commonly mentioned side-effect was weight gain. Instruments were not commonly mentioned in the dataset, but manifested as discussion on what blood glucose monitoring devices and test strips are the best and where to buy them. There were no mentions of diabetes-related apps or websites.

Both Social Support and Social Regulation were markedly connected to Lifestyle, indicating instances of members discussing the validity of a lifestyle-related issue (e.g., particular food or ingredient) and coming to a consensus about it. Explicit mentions of Satisfaction or Dissatisfaction with a conventional doctor, pharmaceutical, or the healthcare system in general were uncommon and exhibited only weak connections to other codes.

Group 1's network structure closely resembles the mean network of the entire dataset, with strong connections among GiveHelp, Symptoms, and Pharma, as well as Lifestyle, Social Support, and Social Regulation. Group 2 members, on the other hand, were more focused on providing help regarding Lifestyle issues and also Sharing about those. There was a significant difference in the discourse of the two groups, mainly driven by the strong connection between GiveHelp and Pharma in group 1. 

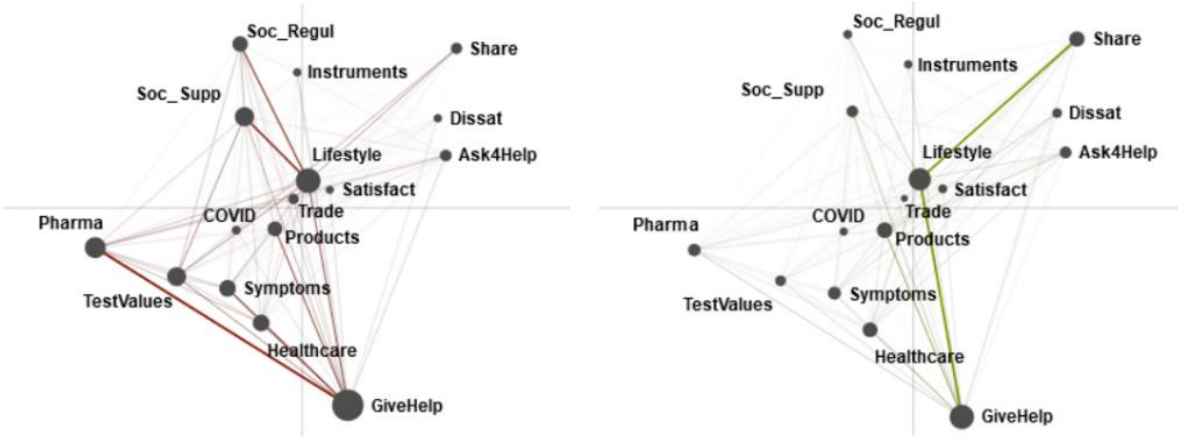

Figure 2. Mean epistemic networks for diabetes self-help group 1 (red, left) and group 2 (green, right) showing the weighted structure of connections among Activity and Content codes. The thickness of the edges (lines) indicates the relative frequency of co-occurrence between each pair of codes; the size of the nodes (black circles) indicates the relative frequency of each code within that group.

We also inspected differences according to year (2020 vs. 2021), as the COVID vaccine was introduced in early 2021, presenting a possible shift in DOC discourse. In 2020, group 1 participants were Sharing Lifestyle and TestValues-related information, but Asking for Help or Giving Help was not prominent at all. Participants were more engaged with discussing various pharmaceuticals and diabetes-related symptoms. In 2021 providing help for group 1 members became very prominent regarding Lifestyle and Pharma, as well as Symptoms. Manifestations of the code COVID (discussions on e.g., vaccines and risk) were much more frequent in 2020 than the following year. Group 2 retained the same basic network structure in both years, with most dominant co-occurrences among GiveHelp, Lifestyle, and Share. The 2021 mean network is slightly more connected, indicating that more threads contained a wider array of codes (e.g., Pharma, Healthcare, Symptoms). 


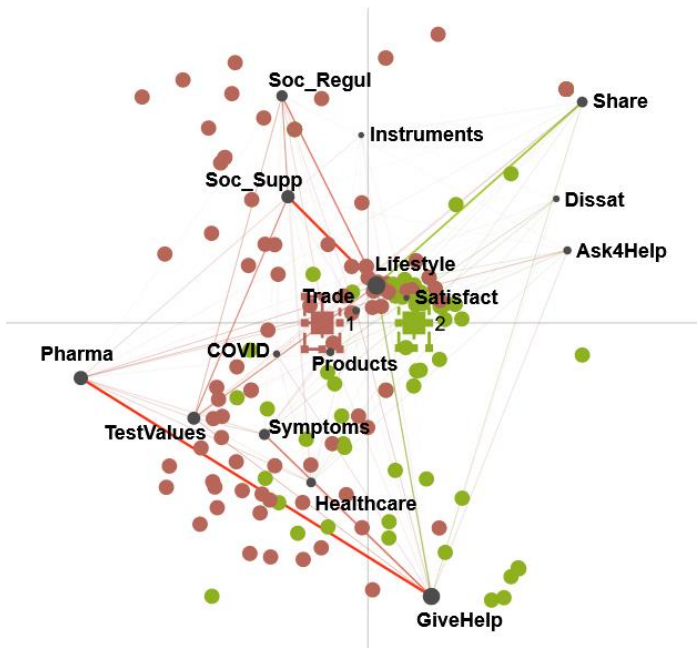

Figure 3. Difference graph showing the subtracted mean networks for both groups. The thickness and saturation of each line indicates the relative difference between the two groups: red lines indicate connections with higher relative frequencies among group 1 members, and green lines indicate connections with higher relative frequencies among group 2 members. The points show the network locations (ENA scores) of each group 1 member (red points) and group 2 member (green points). The colored squares are the mean network locations (mean ENA scores) of each group, and the dashed lines around the means represent the $95 \%$ confidence intervals on each dimension.

\subsection{Critical Facets of Group Discourse}

Initiation and Guidance. One key characteristic of self-help group discourse was experienced PWD guiding newly diagnosed patients regarding basic illness- and self-care related information. Such mentorship was given, for example, on how insulin works and why it is commonly associated with weight gain: "Insulin is the most potent weightgain hormone [...] because it lowers sugar, but if you don't use it immediately, you don't build muscle, it gets stored in fat. Insulin is a growth factor for the body" (tid 39). Also, mentorship emerged concerning the differences between types of insulin: "The active ingredient is the same in both, but there's a difference in absorption. XR indicates that it's absorbed over a long period, so not a big amount all at once, but gradually. It usually has less side-effects than metformin, but even those decrease over time" (tid 70). Not only side-effects are discussed in such a configuration, but symptoms as well: a newly diagnosed patient posted that they had received insulin from the physician and was told to "stay on a strict diet", yet they stated they were experiencing dizziness. An "older" patient replied by telling the newcomer that this is "totally normal" and that her "body is used to higher blood glucose levels and needs time to acclimatize" (tid 64). Diet and nutrition signified another domain where inter-member guidance was frequently provided, e.g.: "Things that are absorbed slowly, like whole-grain, rye, seeds, are recommended for diabetics. If it gets absorbed slowly, that obviously means that your blood sugar goes down more slowly. If the daily $\mathrm{CH}$ intake is low, then the liver 
produces glucose from the stored $\mathrm{CH}$ and releases it into the blood stream, so for a treated patient this may mean a higher glucose level in the morning. Make sure the daily $\mathrm{CH}$ intake is regular and is not lower than, but is equal to the allowed amount. In my case, it's 160 gr." (tid 61). Discussion on diet and nutrition was salient in the data.

Illness Intricacies. Members exchanged information on a variety of issues that may not arise in conventional care settings, such as: the effects of time differences and daylight savings on meal times, travel advice, alternative spots to administer insulin and how often one should shift injection location, whether it is safe to use insulin past its expiration date, what a "good" pre-breakfast glucose level is, and why glucose levels fluctuate despite adhering to conventional recommendations. These issues were not only addressed, but responded to promptly by group members with first-hand experience. Despite it being infrequent, a crucial aspect of group discourse involved members asking others for insulin or offering up their own unused insulin. In some instances, members were left without insulin when they reportedly could not reach their provider, on other occasions, members were prescribed different medication and were left with a surplus of the previous drug. These initiatives were posted publicly but then negotiated privately among individuals involved in the trade. The onset of COVID introduced a new topic of discourse among group members that, due to many reasons, may not have been easily discussed with their provider, namely: which vaccine they were offered by their GP and which they prefer, whether or not to get a vaccine at all, how great of a risk diabetes presents in contracting COVID and complications that may arise. Finally, first-hand experience was exchanged regarding blood glucose meters (specific brands and their advantages), complimentary therapies (products and home remedies), and diabetes-friendly groceries (specific brands and locations to buy).

Social Support and Regulation. A vital feature of group discourse was ensuring a sense of support and community to those sharing problems, questions, or experiences. Social support manifested in encouraging others in continuing their diet, congratulating individuals on their adequate glucose levels, and providing positive feedback on shared experiences, test values, recipes, and external resources (links to diabetes-related information). Social support was often accompanied by social moderation, especially when more contestable statements were made; strong opinions emerged on COVID vaccines, specific foods, and determining adequate glucose levels. For example, one member posted a picture of a meal and asked "What do you think of a yummy dinner like this?" Some responded with positive feedback, such as "Looks good!" and "Give me some!" or "Rather for lunch [not dinner]!", others were more openly opposed: "Don't recommend this for diabetics" and "Sure, if you want a CH of 8 in the morning" (tid 72). When the validity of a claim was contested, it was usually negotiated until a group consensus seemed to emerge. In some cases, this was more easily accomplished than others, such as in the case of a disputed glucose level: the comment "Don't try to tell a new diabetic that a glucose level of 10 is normal" was followed by "But I was told that it shouldn't be higher than 10 and if so, I'm good." The thread ended with "It's not good; they can't get better if they are misinformed" (tid 100). 


\section{Discussion}

We aimed to map the content and structure of discourse in diabetes-related Facebook groups in Hungary, and through juxtaposing our findings with available eHealth tool functionalities, understand what is it that eHealth tools may be lacking, but online support groups are continuing to provide to patients.

In a review of 15 DOC studies, Oser et al. compile needs, which members of such patient groups exhibited and the observed outcomes of having these needs met [2]. The authors report a need to receive and to provide psychosocial, technical, informational, age-specific peer support with a strong focus on diabetes self-management. Oser et al. emphasize that more experienced PWD may feel the need to provide the same kind of support they themselves received earlier on in their illness trajectory, which is also supported by Greene et al. [2, 18]. Expressing gratitude, giving and receiving encouragement is salient across cultures and age-groups [19]; providing help and social support were very prominent in our data as well. Asking for help was less dominant in our networks, indicating that a single request for information or feedback was usually met with many responses providing support. There was a marked need to share information regarding symptoms and experiences, and for those to be normalized or validated by other members. Yet, the majority of sharing and acts of providing help were concerning lifestyle, notably, diet. In a study by Stellefson et al., 30\% of all posts were recipes, which the authors argue build a greater sense of community [1].

Due to the fact that diabetes patients denote a high-risk population for contracting SARS-CoV-2 [20], we were expecting COVID to be a salient topic within our data. Despite this, the code representing this topic did not exhibit a high frequency, and its manifestations were mostly regarding the different vaccines in the time preceding their rollout in Hungary. This indicates that patients were more keen on discussing potential side-effects of various vaccines than disclosing e.g. post-immunization experiences or risk. We found a marked connection between providing help and product-related discourse; many participants were asking about and answering questions on conventional and non-conventional products employable in diabetes care. This connotes a salient topic in most DOC studies $[1,2]$. Greene et al. assert that adverse event reporting occurred frequently in the DOC discourse they examined, such as adverse effects of medications and diet supplements [18]. This was not salient in our sample, but neither was satisfaction (with physicians, healthcare) explicitly stated with a high frequency. Thus, "venting" about challenges and frustrations was not as prominent in our sample as was elsewhere [2].

Apart from being a forum to give and receive peer social support, as well as exchange knowledge and experiences on various products and drugs in diabetes care, patient groups we examined exhibited what Kingod calls "biosociality" [21]. The term was coined for describing acts of peers jointly constructing and shaping self-care knowledge and behaviors based on lived experience. In our sample, code co-occurrences of Social Support and Social Regulation frequently manifested as "biosocial" negotiation regarding specific pieces of disclosed information (glucose levels, nutrition, etc.). Some studies have examined the content and outcomes of biosocial negotiation in terms of validity; for example, Greene et al. state that only $3 \%$ of posts in their sample contained 
inappropriate or unsupported therapeutic information [18]. Despite studies suggesting claims in DOC discussions are by-and-large clinically sound, the potential for misinformation to spread is still present if discourse is left "unsupervised" by medical professionals [18]. Patient-driven discourse leads into domains that do not generally receive attention in conventional care, such as travel-related and other "street-level" disease-management information [18], which signifies a vital part of successful self-care. Additionally, due to the plurality of participants [18] in such support groups (e.g., patients, family members, advertisers, healthcare workers living with diabetes), the potential repository of (mis)information is vast and bears the capacity to address a wide array of issues.

About a dozen apps accessible to Hungarians have been developed for PWD, which are available in Hungarian and English. Most of these apps provide help regarding diet, nutrition, and exercise, as well as measuring and tracking blood glucose levels. Less common, albeit potentially useful functions, include the possibility to contact peers through social media platforms, and, via manually entered data, a warning function to forecast the consequences of a poorly chosen meal on glucose levels. Another useful and increasingly common function is the ability to store and transfer data from a glucose meter to a telephone application, enabling values to be downloaded and presented during medical consultation.

\section{Conclusions}

Building on the finding that giving and receiving peer support, normalization and validation, and "street-level" information, as well as performing biosocial negotiation on a plethora of topics were all prominent features of diabetes support group functioning in our sample, existing eHealth tool functionality could be aggregated and augmented in several ways. For example, it would be beneficial if an application could be integrated with the blood glucose meter, and would automatically record each value. That log could be supplemented with manually entered information on diet (consumed food and drinks as well as their date and time) and insulin use (time and administered dose). These jointly could warn patients when glucose levels are not adequate and provide positive feedback when they are optimal. This app could also have a function for connecting peers based on location, allowing them to share information on e.g., suitable restaurants, exercise programs, and groceries at specific stores in the area. Peer connectivity could also lead to the organization of location-based social events or illness-, symptom-specific social support. Also, open peer-to-peer invitations to exercise programs could enhance participation, as social support is a known driver of adherence to exercise regimes. Furthermore, enabling a way to continue asynchronous chat would be ideal, perhaps on a forum where information could be periodically reviewed by a practitioner. Ideally, the app could be downloaded and used not only by patients, but their physician and/or their dietitian, which, if necessary, would provide them with information on the administered treatment, glucose levels, and nutrition. 


\section{$7 \quad$ Limitations and Future Directions}

Our study had several limitations. If individuals would have been considered cases instead of groups, additional data, namely, demographic information (e.g., age, sex, and level of education) and health literacy measures could have enriched analysis and provided extra dimensions in interpretation. This information was only partly available to us, hence we opted not to include it in the study. This research initiative could have been also been supplemented with a social network analysis to shed more light on how group participants are interacting with each other. We did not measure engagement in this study (e.g., number of likes and shares) because it did not constitute part of our research questions, but a secondary analysis could focus on which pieces of content were most popular and impactful in DOCs. Our investigation did pertain to discourse content though, and we disregarded visual and external content (i.e., pictures, gifs, and links were not included). It is common in social media discourse analyses to limit the scope of data to that displayed on the platform under investigation; we followed this protocol, although, external content could potentially be included in a secondary analysis. In social media analyses, pictures are frequently coded as a type of communication ("post feature"), alongside other general codes such as text and video. As group discourse did not exhibit much variety among types of communication (it consisted almost exclusively of text), we decided not to include these codes in our study. Our future analyses may benefit from empirically proving this observation, but it did not fall under our present aims. Finally, we encountered our greatest limitation at data collection: there were no more public diabetes groups on Facebook apart from the two presently under scrutiny. And, as Stellefson et al. also notes: private groups may exhibit vastly different behavior in terms of thread content and social activities [1]; these remained outside of our investigation due to the fact that this data was not publicly accessible.

\section{Acknowledgements}

This project constitutes part of a larger investigation entitled "E-patients and e-physicians in Hungary: The role and opportunities of digital health solutions in the healthcare system" conducted as part of a grant awarded by the National Research, Development and Innovation Office, Hungary (Grant: FK_20; Duration: 2020.09-2024.09). 


\section{References}

1. Stellefson M, Paige S, Apperson A, Spratt S (2019) Social Media Content Analysis of Public Diabetes Facebook Groups. J Diabetes Sci Technol 13:428-438. https://doi.org/10.1177/1932296819839099

2. Oser TK, Oser SM, Parascando JA, et al (2020) Social Media in the Diabetes Community: a Novel Way to Assess Psychosocial Needs in People with Diabetes and Their Caregivers. Curr Diab Rep 20:10. https://doi.org/10.1007/s11892-020-1294-3

3. Liu NF, Brown AS, Folias AE, et al (2017) Stigma in People With Type 1 or Type 2 Diabetes. Clin Diabetes 35:27-34. https://doi.org/10.2337/cd16-0020

4. Kwan B, Jortberg B, Warman K, et al (2017) Stakeholder engagement in diabetes selfmanagement: Patient preference for peer support and other insights. Family practice 34:. https://doi.org/10.1093/fampra/cmw127

5. Herrero N, Guerrero-Solé F, Mas-Manchón L (2021) Participation of Patients With Type 2 Diabetes in Online Support Groups is Correlated to Lower Levels of Diabetes Self$\begin{array}{lllll}\text { Management. J } & \text { Diabetes Sci Technol }\end{array}$ https://doi.org/10.1177/1932296820909830

6. Gabarron E, Larbi D, Dorronzoro E, et al (2020) Factors Engaging Users of Diabetes Social Media Channels on Facebook, Twitter, and Instagram: Observational Study. J Med Internet Res 22:e21204. https://doi.org/10.2196/21204

7. Mattson M, Hall J (2011) Linking health communication with social support. In: Health as Communication Nexus: A Service Learning Approach. Kendall Hunt Publishing, United States: Dubuque, IA

8. Wong D, Amon KL, Keep M (2019) Desire to Belong Affects Instagram Behavior and Perceived Social Support. Cyberpsychol Behav Soc Netw 22:465-471. https://doi.org/10.1089/cyber.2018.0533

9. Tenderich A, Tenderich B, Barton T, Richards S (2019) What Are PWDs (People With Diabetes) Doing Online? A Netnographic Analysis - PubMed. J Diab Sci Technol 13:187197

10. Zhang Y, He D, Sang Y (2013) Facebook as a platform for health information and communication: a case study of a diabetes group. J Med Syst 37:9942. https://doi.org/10.1007/s10916-013-9942-7

11. Csanadi A, Eagan B, Kollar I, et al (2018) When coding-and-counting is not enough: using epistemic network analysis (ENA) to analyze verbal data in CSCL research. In: International Journal of Computer-Supported Collaborative Learning, 4th ed. pp 419-438

12. Rus HM, Cameron LD (2016) Health Communication in Social Media: Message Features Predicting User Engagement on Diabetes-Related Facebook Pages. Ann Behav Med 50:678-689. https://doi.org/10.1007/s12160-016-9793-9

13. Bowman D, Swiecki Z, Cai Z, et al (2021) The mathematical foundations of epistemic network analysis. In: Advances in Quantitative Ethnography. Springer, Cham Switzerland, pp 91-105

14. Statista (2020) Social media penetration rate in Hungary in 2020, by platform

15. Zörgő S, Peters G (2019) Epistemic Network Analysis for Semi-Structured Interviews and Other Continuous Narratives: Challenges and Insights. In: Advances in Quantitative Ethnography. Communications in Computer and Information Science Series. pp 267-277 
16. Peters G-JY, Crutzen R (2017) Pragmatic nihilism: How a Theory of Nothing can help health psychology progress. Health Psychology Review 11:103-121. https://doi.org/10.1080/17437199.2017.1284015

17. Eagan BR, Rogers B, Serlin R, et al (2017) Can We Rely on IRR? Testing the Assumptions of Inter-Rater Reliability

18. Greene JA, Choudhry NK, Kilabuk E, Shrank WH (2011) Online social networking by patients with diabetes: a qualitative evaluation of communication with Facebook. J Gen Intern Med 26:287-292. https://doi.org/10.1007/s11606-010-1526-3

19. White K, Gebremariam A, Lewis D, et al (2018) Motivations for Participation in an Online Social Media Community for Diabetes. Journal of Diabetes Science and Technology 12:712-718

20. Singh AK, Gupta R, Ghosh A, Misra A (2020) Diabetes in COVID-19: Prevalence, pathophysiology, prognosis and practical considerations. Diabetes Metab Syndr 14:303-310. https://doi.org/10.1016/j.dsx.2020.04.004

21. Kingod N (2020) The tinkering m-patient: Co-constructing knowledge on how to live with type 1 diabetes through Facebook searching and sharing and offline tinkering with selfcare. Health (London) 24:152-168. https://doi.org/10.1177/1363459318800140 